\title{
Formulation and bioequivalence testing of fixed-dose combination orally disintegrating tablets for the treatment of tuberculosis in paediatric population
}

Thomas J. Dennison ${ }^{1}$, Julian C. Smith², Raj K.S. Badhan ${ }^{1}$ and Afzal R. Mohammed ${ }^{1 *}$

${ }^{1}$ Aston School of Pharmacy, Aston University, Birmingham, UK

${ }^{2}$ Faculty of Computing, Engineering and Science, University of South Wales., UK

* Corresponding Author:

18 Afzal R Mohammed

Email: a.u.r.mohammed@aston.ac.uk

Phone: 0121-204 4183 


\begin{abstract}
1 Abstract
Tuberculosis (TB) is believed to affect around 10 million people worldwide. Treatment for TB includes isoniazid and rifampicin, with fixed-dose combination (FDC) recommended for improved patient compliance. Similarly, orally disintegrating tablets (ODTs) are an increasingly popular dosage form that aid compliance since they do not require swallowing. In this study ODTs of isoniazid and rifampicin, either as discrete or FDC doses, were formulated and bioequivalence between single and combination doses compared using in vitro and in silico approaches.

9 Dissolution profiles were compared using FDA advised difference $\left(f_{1}\right)$ and similarity $\left(f_{2}\right)$ testing in biorelevant media. Rifampicin release from FDCs decreased by approximately $15 \%$ in fed-state media (failed $f_{1}$ and $f_{2}$ ), which was attributed to enhanced rifampicin degradation in the presence of isoniazid at lower $\mathrm{pH}$. Apparent permeability $\left(\mathrm{P}_{\mathrm{app}}\right)$ values derived from Caco-2 transport studies were included alongside dissolution results into a physiologically based pharmacokinetic (PBPK) model, to simulate in vivo bioavailability in healthy subjects. Models showed no difference in bioavailability between formulations or dosing (fasted or fed) state, despite the failures in dissolution-based bioequivalence testing, highlighting shortcomings in $\mathrm{f}_{1}$ and $\mathrm{f}_{2}$ assessment and the strength of PBPK models.
\end{abstract}




\section{Introduction}

Recognised as one of humankind's oldest diseases, with evidence of cases dating back more than 5000 years ${ }^{1}$, tuberculosis (TB) remains a major cause of morbidity and mortality. Today there are an estimated 9.6 million TB cases worldwide, with the disease claiming 1.5 million deaths in 2014 alone ${ }^{2}$. Since 2000 the incidence of TB has fallen by $18 \%$, at an average rate of $1.5 \%$ per year, with effective treatment within this time frame saving an estimated 43 million lives ${ }^{2}$.

TB is an infectious disease caused by the aerobic bacterium Mycobacterium tuberculosis (MTB). Transmission occurs through aerosolisation of the bacterium into droplet nuclei by coughing, sneezing or talking ${ }^{3}$. Inhalation of the organism into the alveoli leads to respiratory infection, that if spreads, causes extrapulmonary tuberculosis, which can involve any organ system in the body ${ }^{4}$. Pulmonary tuberculosis, the most common presentation, is avoided in most cases of exposure through mucociliary clearance ${ }^{5}$, or failing that through the successful activity of phagocytic alveolar macrophages, resulting in symptomless latent tuberculosis ${ }^{6}$. Around $5 \%$ of TB infections progress to the active form of the disease within two years, with about $10 \%$ of latent cases reactivating at some point later in life ${ }^{3}$. TB outcome is dependent on a multitude of factors, most prominent of which is the immunocompetence of the individual, which itself depends on various intrinsic and extrinsic factors such as the hosts genetics and nutritional state, respectively ${ }^{7,8}$.

Clinical manifestation of TB depends on the site of infection. Pulmonary TB, historically referred to as consumption or pthisis, classically manifests as severe wasting ${ }^{8}$, as well as cough, haemoptysis, chest pain, dyspnoea, malaise, fatigue, low-level fever and night sweats 9 . Extrapulmonary TB can include the same symptoms as pulmonary TB, with a wide range of additional symptoms based upon the site of infection, such as meningitis (CNS), lymphadenitis (lymphatic), arthritis (skeletal) and haematuria (renal) ${ }^{10}$.

Various social, environmental and biological risk factors determine the risk of TB contraction. Risks for infection and progression to disease are distinctly different; infection risk involves extrinsic factors including social and behavioural risks (alcohol, smoking and pollution), source infectiousness and proximity (including overcrowding and length of exposure), whereas risk of progression to disease is endogenous to the host ${ }^{11}$. Immunosuppressive conditions accelerate progression to active disease, with HIV being especially potent ${ }^{12}$. Impaired immune response as a result of malnutrition is also known to increase the risk of TB ${ }^{13}$, whilst a strong socioeconomic association with the disease exists, with the poorest experiencing the greatest risk ${ }^{14}$. Children also present an increased susceptibility to TB development, which is greater still before the age of 2 and after age $10{ }^{15}$. Other risk factors for progression to disease include diabetes, alcohol, smoking and indoor air pollution ${ }^{11}$.

Isoniazid and rifampicin form the basis of front-line treatment for $\mathrm{TB}^{2}$, with both drugs included in the WHO Model List of Essential Medicines and Essential Medicines for Children. Isoniazid (BCS class $\mathrm{I} / \mathrm{III}{ }^{16}$ ) is a pro-drug that requires activation by catalase-peroxidase enzyme (KatG), which is endogenous to MTB ${ }^{17}$. The drug inhibits the synthesis of mycolic acids, essential components of the bacterial cell wall and at therapeutic doses is bactericidal against actively growing intra and extra cellular MTB ${ }^{18}$. Rifampicin (BCS class $\mathrm{II}^{19}$ ) also displays a bactericidal effect on MTB, by inhibition of transcription through high-affinity binding to the $\beta$-subunit of bacterial DNA-dependent RNA polymerase ${ }^{20}$. Rifampicin is highly effective against TBM through its ability to readily diffuse into tissues, cells and bacteria ${ }^{21}$. The tendency of rifampicin to degrade substantially when combined with isoniazid in acidic media is a well-recognised complication when considering combination of the two drugs in solid oral-dosage forms ${ }^{22}$.

The first-line recommended oral drug regimen for treatment of drug susceptible TB involves isoniazid, rifampicin, pyrazinamide and ethambutol for 2 months, followed by isoniazid and rifampicin for 4 months, with the regimen altering due to drug or multi-drug resistance ${ }^{23}$. Treatment for extrapulmonary TB does not differ, except in some cases where duration of therapy is extended 24. Recommended doses for treatment of children differ compared to adults. Fixed-dose combinations (FDCs) are recommended for TB treatment of both adults and children ${ }^{23}$, however FDCs currently on the market do not correspond to appropriate doses for children ${ }^{24}$. FDCs for TB treatment have not been shown to alter efficacy, drug resistance or adverse effects or events when compared to single-dose ${ }^{24}$. Furthermore, whilst FDCs have not provided evidence for 
improvement of treatment outcomes, their use simplifies TB therapy, with some evidence for an increase in patient satisfaction ${ }^{25}$.

Orally disintegrating tablets (ODTs) are an increasingly popular dosage form that improve compliance for patients with dysphagia, a difficulty swallowing, particularly prevalent in paediatric and geriatric populations, institutionalised and psychiatric patients and sufferers of nausea and vomiting ${ }^{26}$. ODTs are designed to rapidly disintegrate in contact with saliva within the oral cavity, removing the need for swallowing and coadministration with water. Market studies have shown ODTs to be popular amongst patients, with over $50 \%$ preferring them to other dosage forms (such as regular tablets and liquids) and $70 \%$ of consumers declaring they would request ODTs from their physician ${ }^{27}$.

In order for a new generic formulation to be approved it needs to demonstrate bioequivalence with a reference branded product. A bioequivalent drug will display comparable bioavailability and thus in vivo performance (efficacy and safety) ${ }^{28}$. Bioequivalence can be assumed in the absence of clinical trials, if there is no significant difference in the rate and extent to which the active pharmaceutical ingredient (API) becomes available within the systemic circulation, when compared with the reference product ${ }^{29}$. Bioequivalence testing may also be applied in the assessment of FDCs ${ }^{28}$. For immediate release formulations bioequivalence can be determined by comparison of in vitro dissolution profiles using FDA recommended difference factor $\left(\mathrm{f}_{1}\right)$ and similarity factor $\left(\mathrm{f}_{2}\right)$ testing, for biowaiver applications ${ }^{29}$. Comparison testing is not deemed necessary if test products display greater than $85 \%$ dissolution within 15 mins, given that the API falls within BCS class I or III (although class III carries stricter requirements) ${ }^{28}$. The extension of biowaivers to BCS class II compounds is a topic of much discussion ${ }^{30}$.

Pharmacokinetic modelling and simulation has become an established tool over the past 20 years to predict drug pharmacokinetics in humans and assess the effect of intrinsic and extrinsic factors on drug exposure. Physiologically based pharmacokinetic (PBPK) models define tissues and organs as compartments, with parameters based upon decades of knowledge of body fluid dynamics. PBPK models consider ADME processes throughout all compartments to estimate the pharmacokinetic profile of a drug at a target tissue or organ ${ }^{31}$. As such, PBPK models have become a powerful tool for the prediction of oral drug absorption through integration of common in vitro drug-specific information, alongside a variety of physiological descriptions of the population groups (e.g. age, weight, height, tissue perfusion, drug metabolising enzyme abundance and ontogeny) ${ }^{32}$. PBPK modelling is often exploited for prediction of oral drug absorption and to study formulation changes ${ }^{33}$ or FDCs ${ }^{34}$. There is also an effort to apply PBPK modelling to predict the bioequivalence of new generic formulations with reference drugs ${ }^{35}$.

An FDC ODT for isoniazid and rifampicin could potentially improve patient compliance and be particularly beneficial in developing areas with little to no access to water. The use of a paediatric relevant dose would be valuable given the current lack of support and the widely reported and supported applicability of ODTs to enhance compliance in paediatric populations ${ }^{36}$. Similarly, improved clinical outcomes from FDCs, due primarily to improved adherence as a result of reduced pill burden, are well documented ${ }^{37}$.

This work demonstrates the ability of PBPK modelling and clinical trial simulations to overcome the challenge of drug testing in paediatric populations. Specifically, this study focuses on the development of isoniazid and rifampicin single and FDC ODT formulations at paediatrically relevant doses. In vitro drug dissolution and permeability data was used to predict drug pharmacokinetics for in silico models, in order to investigate API bioequivalence between single and fixed-dose formulations. 


\section{Materials}

Isoniazid and rifampicin were purchased from Molekula Ltd (UK). Pearlitol® Flash (mannitol-starch copolymer) was obtained from Roquette Pharma (France), whilst Avicel PH-102 micro-crystalline cellulose (MCC) and sodium stearyl fumarate (SSF) were purchased from FMC BioPolymer (USA).

Biorelevant FaSSIF/FeSSIF/FaSSGF Instant Powder was purchased from biorelevant.com (UK). Sodium hydroxide, sodium chloride, sodium phosphate and glacial acetic acid for biorelevant media were obtained from Sigma-Aldrich (UK). Acetonitrile (ACN) and methanol (HPLC-grade) were obtained from Fisher Scientific (UK).

For cell culture media DMEM was purchased from Lonza (UK), fetal bovine serum (FBS), gentamicin (10 mg/ml), Fungizone (amphotericin B $250 \mu \mathrm{g} / \mathrm{ml}$ ), HBSS and penicillin/streptomycin $(10,000 \mathrm{U} / \mathrm{ml})$ were all purchased from Gibco (Thermo Fischer Scientific, UK). Trypsin-EDTA solution $(0.25 \%)$ was procured from Sigma-Aldrich (UK).

Tablet production

Direct compression of tablets $(500 \mathrm{mg})$ at a compaction force of 2.2 tons was performed on an Atlas T8 automatic press (SPECAC, UK), using a $13 \mathrm{~mm}$ round, flat-faced die. Tablets were produced under ambient conditions.

\section{Disintegration testing}

Disintegration testing was performed in accordance with US pharmacopeia monograph ([701] disintegration). An Erweka ZT3, Appartebau, GMBH (Germany) was used as disintegration apparatus and $800 \mathrm{ml}$ distilled water maintained at $37^{\circ} \mathrm{C}$ was used as the disintegration media. Tablets were measured individually by placing in the basket rack and the time taken for the tablets to disintegrate without leaving any solid residue in the rack, recorded. Disintegration time was measured in triplicate.

\section{Friability}

Tablet friability was determined on 6 tablets using an F2 friability tester (Sotax, Switzerland). Tablets were placed inside the drum and rotated at $25 \mathrm{rpm}$ for a total of 100 revolutions. Dust was removed pre and post testing to remove excess powder that would contribute to tablet mass. Friability was calculated and expressed as \% tablet weight loss from initial tablet weight.

Tablet hardness

A Tablet Hardness Tester TBF1000 (Copley Scientific, UK) was used to measure the radial crushing strength (hardness) of tablets in triplicate.

\section{High performance liquid chromatography (HPLC)}

HPLC was performed on an Agilent 1260 series (Agilent Technologies, USA), comprising a quarternary pump, Infinity VWD and autosampler. Analysis was conducted on a reversed-phase Gemini C18, 150 x $4.6 \mathrm{~mm}, 110 \AA$, 5 $\mu \mathrm{m}$ column (Phenomenex, UK). Protocols were developed, calibrated and validated for both isoniazid and rifampicin alone and in combination.

Separations were achieved using either (Type 1) $\mathrm{H}_{2} \mathrm{O}, 0.1 \%$ (v/v) TEA, $0.1 \%$ (v/v) TFA or ACN at different ratios as the mobile phase. Ascorbic acid $(0.5 \mathrm{mg} / \mathrm{ml})$ was included as an antioxidant to prevent rifampicin degradation ${ }^{38}$. Isoniazid separation was performed with an isocratic mobile phase of $\mathrm{H}_{2} \mathrm{O}$ : ACN $(90: 10 \mathrm{v} / \mathrm{v})$, a flow rate of $1 \mathrm{ml} / \mathrm{min}$ and a wavelength of $254 \mathrm{~nm}$. Rifampicin 
separation was achieved using an isocratic mobile phase of TFA: ACN (45:55 v/v), a flow rate of

$21 \mathrm{ml} / \mathrm{min}$ and a wavelength of $254 \mathrm{~nm}$. Separation of isoniazid and rifampicin in combination required a mobile phase of TEA: ACN delivered at a gradient (95:5 to 20:80 v/v), with a flow rate of $1 \mathrm{ml} / \mathrm{min}$ and a wavelength of $254 \mathrm{~nm}$. An injection volume of $20 \mu \mathrm{l}$ was used throughout.

HPLC method validation involved assessment of precision through intra-day variation, accuracy by multilevel recovery studies, instrument precision, linearity and limit of detection and quantification (LOD and LOQ). Stock solutions $(1 \mathrm{mg} / \mathrm{ml})$ of each drug were prepared in mobile phase from which dilutions and subsequently two-fold serial dilutions were prepared to form a calibration curve.

\section{Dissolution testing}

API dissolution from ODTs in $900 \mathrm{ml}$ biorelevant media (FaSSIF/FeSSIF/FaSSGF instant powder, biorelevant.com, UK) was tested in both fasted state simulated intestinal fluid (FaSSIF) and fed state simulated intestinal fluid (FeSSIF), at pH 6.5 and 5 respectively and maintained at $37^{\circ} \mathrm{C}$. An ERWEKA DT 600 USP 2 paddle apparatus (Germany) was used at a paddle speed of $50 \mathrm{rpm}{ }^{39}$. $5 \mathrm{ml}$ samples were taken over $2 \mathrm{~h}$, replacing with $5 \mathrm{ml}$ fresh media to simulate sink conditions. API dissolution was measured using HPLC and corrected for \% dose dissolved.

\section{Cell culture}

Prior to seeding, cells were trypsinised $(2.5 \mathrm{ml})$ from $75-\mathrm{cm}^{2}$ cell culture flasks (Corning, USA) on which they had been grown (80\% confluence), after washing with HBSS. Caco-2 cells (passage 54-58) were seeded onto Transwell (Corning, USA) semi-permeable membrane supports (12-well, $1.12 \mathrm{~cm}^{2}, 0.4 \mu \mathrm{m}$ pore size) at a density of $8 \times 10^{4} \mathrm{cells} / \mathrm{cm}^{2}$. Cells were maintained in Dulbecco's modified Eagle's minimal essential medium (DMEM) containing L-glutamine (4 mM) and glucose $(4.5 \mathrm{mg} / \mathrm{ml})$, and supplemented with ( $\mathrm{v} / \mathrm{v}) 10 \%$ fetal bovine serum, $1 \%$ penicillin/streptomycin, $1 \%$ non-essential amino acids, amphotericin B $(0.5 \mu \mathrm{g} / \mathrm{ml})$ and gentamicin $(20 \mu \mathrm{g} / \mathrm{ml})$. Media was changed every 2-3 days and transwells cultured at $37^{\circ} \mathrm{C}, 5 \% \mathrm{CO}_{2}$ for 21 days, after which transport studies were performed.

\section{Caco-2 transport studies}

Caco-2 cells were purchased from the European Collection of Authenticated Cell Cultures (ECACC) via Public Health England. Caco-2 monolayers were used for transport studies between 21 and 24 days post-seeding. Drug absorption through Caco-2 monolayers was measured for isoniazid and rifampicin alone and in combination in both the apical to basolateral $(A-B)$ and basolateral to apical (B-A) directions $(n=3)$. Transport studies were carried out in DMEM $\left(37^{\circ} \mathrm{C}\right)$ containing $10 \mathrm{mM}$ HEPES ( $\mathrm{pH} 7.4$ ), with $0.5 \mathrm{ml}$ and $1.5 \mathrm{ml}$ in the A and B compartments, respectively. Samples of $100 \mu \mathrm{l}$ were removed from the A side and $200 \mu \mathrm{l}$ from the B side at time points over $2 \mathrm{~h}$, replacing with fresh pre-warmed media $\left(37^{\circ} \mathrm{C}\right)$ to mimic sink conditions. For mass balance, samples were taken from the donor compartments at $t=0$ and $t=120$ mins.

Isoniazid was administered at a concentration of $20 \mu \mathrm{g} / \mathrm{ml}$ and rifampicin at a concentration of 30 $\mu \mathrm{g} / \mathrm{ml}$. Concentrations used were comfortably within or below previously reported well tolerated concentration ranges for both isoniazid and rifampicin ${ }^{40}$. Cultures were maintained at $37^{\circ} \mathrm{C}$ and $5 \% \mathrm{CO}_{2}$ throughout the experiment. Samples were analysed by HPLC and apparent permeability (Papp) values were calculated using equation:

$$
P_{a p p}=(d Q / d t) /\left(C_{0} \times A\right)
$$

Where $d Q / d t$ is the mass transfer rate of the compound from the donor to the receiver compartment, $\mathrm{C}_{0}$ is the initial concentration in the donor chamber and $\mathrm{A}$ is the monolayer surface area $\left(\mathrm{cm}^{2}\right)$. 


\section{Clinical trials simulation}

The population-based clinical trials simulator Simcyp (V14) (Certara, USA) was used to simulate the plasma concentration of isoniazid and rifampicin from single API and FDC formulations. Default parameter values for creating a North European Caucasian population were selected ${ }^{41}$.

\section{Compound data}

Physicochemical information for each API was collated from the literature used to develop compound files (Table 8). Simulations were performed using a minimal-PBPK model. Where uncertainty arose regarding the precise value of compound data parameters, parameter estimation was conducted using the Parameter Estimation Module to optimize parameter values. The ADAM model ${ }^{42}$ was assumed for all simulations and the dissolution profile for each formulation (single and FDC) in FaSSIF and FeSSIF was utilised.

\section{Clinical studies}

The optimization and validation of the PBPK model was conducted using clinical study results reported in healthy adult subjects. For isoniazid: study 1 included a total dose of $300 \mathrm{mg}$ dosed to 18 healthy volunteers (18-55 years old) ${ }^{43}$; study 2 included a total dose of $300 \mathrm{mg}$ dosed to 22 healthy volunteers ${ }^{44}$; study 3 included a total dose of $300 \mathrm{mg}$ dosed to 20 healthy volunteers (23 \pm 1.8 years old) 45 ; study 4 included a total dose of $300 \mathrm{mg}$ dosed to 18 healthy volunteers (36.4 \pm 10.6 years old ${ }^{46}$. Studies 1 and 2 were used for model development and studies 3 and 4 utilized for validation.

For rifampicin: study 1 included a total dose of $600 \mathrm{mg}$ dosed to 18 healthy volunteers (18-55 years old ${ }^{43}$; study 2 included a total dose of $600 \mathrm{mg}$ dosed to 20 healthy volunteers $(23 \pm 1.8$ years old) ${ }^{45}$; study 3 included a total dose of $600 \mathrm{mg}$ dosed to 18 healthy volunteers $(36.4 \pm 10.6$ years old) ${ }^{46}$; study 4 included a total dose of $600 \mathrm{mg}$ dosed to 22 healthy volunteers ${ }^{44}$. Studies 1 and 2 were used for model development and studies 3 and 4 utilized for validation.

Raw data from published human trial plasma concentration profiles was extracted using WebPlotDigitizer $3.10^{47}$ and, where necessary, parameter estimation was conducted using the validation clinical datasets.

Predictions of API plasma pharmacokinetic profiles were simulated following the oral administration of a single immediate release solid dosage form of $50 \mathrm{mg}$ (isoniazid) and $75 \mathrm{mg}$ (rifampicin) dose over a $24 \mathrm{hr}$ period.

To assess the impact of $A B C B 1$ active efflux on rifampicin fraction dose absorbed (fa), we conducted a local sensitivity analysis by varying ABCB1 active transport clearance (CLtrans) $(0.1$ to $100 \mu \mathrm{L} / \mathrm{min} / \mathrm{pmol})$ and Papp $\left(0.1-100 \times 10^{-6} \mathrm{~cm} / \mathrm{s}\right)$, then assessing the resulting impact on fa.

\section{Statistical analysis}

GraphPad PRISM software version 6.01 (USA) was used for data analysis. Ordinary one-way ANOVA was used with Tukey's multiple comparisons test to analyze data for tablet characterization. Unpaired two-tailed t-test was used to determine statistical differences between data sets for pharmacokinetic parameters.

Differences between dissolution profiles of APIs in single dose (reference) and combination (test) were assessed using $f_{1}$ and $f_{2}$ difference and similarity factor testing, using the equations ${ }^{48}$ :

$$
f_{1}=\left(\left[\sum_{t=1}^{n}\left|R_{t}-T_{t}\right|\right] /\left[\sum_{t=1}^{n} R_{t}\right]\right) * 100
$$

$$
f_{2}=50 * \log \left(\left[1+(1 / n) \sum_{t=1}^{n}\left(R_{t}-T_{t}\right)^{2}\right]^{-0.5} * 100\right)
$$


2 Where $R_{t}$ and $T_{t}$ are the \% drug dissolved value at each time point for the reference and test 3 product respectively and $\mathrm{n}$ is the number of time points. 


\section{ODT development}

3

4

An ODT formulation for rifampicin and isoniazid both alone and in combination was developed, with the requirement that tablets were mechanically robust whilst maintaining rapid disintegration. Round flat faced tablets $(500 \mathrm{mg}$ ) were produced by direct compression. In order to isolate the effect of combination of APIs, the number of excipients used was kept at a minimum. The formulation consisted of API alongside Na stearyl fumerate (SSF, $0.5 \% \mathrm{w} / \mathrm{w}$ ) as a lubricant and Pearlitol as a diluent. Compaction forces were applied at a range of 1-2 T, with hardness values acceptable $(>60 \mathrm{~N})$ from a compaction force of $1.2 \mathrm{~T}$ and above. Friability values at all compaction forces were high $(>1 \%)$, with tablets compressed at and below $1.2 \mathrm{~T}$ unable to withstand friability testing. Disintegration times at all compaction forces were within $30 \mathrm{~s}(18-21 \mathrm{~s})$, as recommended by the FDA for ODTs ${ }^{49}$, with no significant effect $(p>0.05)$ on disintegration with changes in compaction force. At $2 \mathrm{~T}$ compaction force tablet hardness peaked at $100.17 \pm 7.97 \mathrm{~N}$ and friability dropped to $1.97 \%$. Increasing SSF to $1.5 \% \mathrm{w} / \mathrm{w}$ ensured improved lubricant ability whilst maintaining high hardness and a low disintegration time.

To combat high friability MCC was included as a binder ${ }^{50}$. Addition of MCC up to $15 \% \mathrm{w} / \mathrm{w}$ increased hardness ( $p>0.01$ ), compared to $5 \%$ and $10 \%$, to $119.50 \pm 3.90 \mathrm{~N}$, whilst lowering friability and maintaining rapid disintegration. MCC has excellent binding properties due to its plastic deformation, maximising interparticulate bonding ${ }^{50}$ and hydrogen bond formation between adjacent molecules ${ }^{51}$, whilst mechanical interlocking has also been proposed as a mechanism ${ }^{52}$. The high intraparticle porosity of MCC promotes rapid penetration of water through capillary action and is responsible for its ability to enhance disintegration ${ }^{50}$. Raising compaction force to $2.2 \mathrm{~T}$ lowered tablet friability to $1.10-0.85 \%$, maintained rapid disintegration and raised hardness to as high as $151.17 \pm 4.48 \mathrm{~N}$. Formulation composition is shown in Table 1 and characterisation of formulations is shown in Table 2.

\section{HPLC protocol validation}

Linearity test solutions were prepared from stocks at six concentrations ranging from 100 to 1.5625 $\mu \mathrm{g} / \mathrm{ml}$. Validation of protocols by intraday studies for isoniazid, rifampicin and isoniazid/rifampicin combination are shown in Table 3. Instrument precision, tested for by six consecutive injections of the same sample $(100 \mu \mathrm{g} / \mathrm{ml})$, ranged from $0.08 \%$ to $0.87 \%$. All protocols showed good method accuracy and precision. Method accuracy is demonstrated by multilevel recovery, ranging from $100 \mu \mathrm{g} / \mathrm{ml}$ to $6.25 \mu \mathrm{g} / \mathrm{ml}$. Accurate recovery was exhibited at each concentration for both APIs, ranging from $98.03 \%$ to $101.98 \%$, with mean recovery values shown. Relative standard deviation (RSD) values representing intraday precision for isoniazid, rifampicin and isoniazid/rifampicin were low, ranging from 0.51 to $2.40 \%$, with mean values displayed. LOQ and LOD values for isoniazid and rifampicin alone were at or below 0.80 and $0.24 \mu \mathrm{g} / \mathrm{ml}$, respectively. LOQ and LOD values for isoniazid in combination were lower still, whilst rifampicin in combination showed the greatest LOQ and LOD of 1.18 and $0.36 \mu \mathrm{g} / \mathrm{ml}$, respectively.

\section{Dissolution}

Drug release from ODTs was tested in FaSSIF and FeSSIF media (Table 4). Rapid and complete isoniazid dissolution from single dose (99.24\%) and FDC (100.65\%) in FaSSIF (Figure 1) was observed. Difference testing showed dissolution profiles were equivalent $\left(f_{1}=14.17\right)$ however similarity testing indicated differences between both profiles $\left(\mathfrak{f}_{2}=32.79\right)$. Despite this, isoniazid dissolution exceeded 85\% within 15 mins. In FeSSIF (Figure 2) similar drug release profiles for isoniazid are seen from both single and FDC formulations, with dissolution peaking at $100.12 \%$ and $101.52 \%$, respectively and both formulations exceeding $85 \%$ dissolution by 5 mins. Values for $f_{1}$ and $f_{2}$ testing show no difference between the two dissolution profiles.

Rifampicin dissolution from single and FDC formulations in FaSSIF (Figure 3) was comparable based on $f_{1}$ and $f_{2}$ testing, with complete dissolution of $100.63 \%$ from single dose, whilst dissolution from FDC peaked at $91.91 \%$. Dissolution profiles for rifampicin from single and FDC in FeSSIF (Figure 4) were deemed different, failing $f_{1}$ and $f_{2}$ testing. Rifampicin was rapidly 
released from single dose, showing $>85 \%$ dissolution by 5 mins, peaking at $98.26 \%$, however in combination rifampicin release was retarded, with a maximum dissolution after $1 \mathrm{~h}$ of $85.32 \%$. This observed drop in dissolution is likely a result of degradation, given the complete release seen from the single dose formulation and the well documented enhanced degradation of rifampicin in the presence of isoniazid under acidic conditions, in this instance $\mathrm{pH} 5$.

\section{Permeability studies}

Transepithelial electrical resistance (TEER) values for Caco-2 cells over 21 days plateau from day 18 , showing a resistance of $1351.1 \pm 88.6 \Omega \cdot \mathrm{cm}^{2}$ by day 21 post-seeding. Isoniazid and rifampicin transport across Caco-2 monolayers alone and in combination was measured in A-B and B-A directions. $P_{\text {app }}$ values are summarised for each drug and drug combination in Table 5.

Isoniazid was readily absorbed across Caco-2 monolayers from both A-B and B-A directions, exhibiting an efflux ratio of 1.18 indicating passive diffusion. Similar permeability was displayed for isoniazid in combination with rifampicin, with an efflux ratio of 1.19. Rifampicin Papp values suggested active efflux of the compound, with efflux ratio values of 4.33 and 2.61 from single and combination respectively. Active efflux of rifampicin across Caco-2 monolayers has previously been indicated ${ }^{53}$.

\section{Clinical trials simulation}

The initial simulation of the kinetics of isoniazid (derived from data presented in Table 8) were used to optimize the human jejenum effective permeability and volume of distribution at steady state ( $P_{\text {eff }}$ and $V_{\text {ss, }}$, respectively) from clinical data sets 1 and 2 for each API. Peff describes a prediction of human absorption rate constants (ka), whereas $V_{s s}$ values describe a conversion factor (mass to concentration) and the tissue distribution of the API. Optimized $P_{\text {eff }}$ and $V_{\text {ss }}$ were estimated as $10.23 \times 10^{-4} \mathrm{~cm} / \mathrm{s}$ and $0.63 \mathrm{~L} / \mathrm{kg}$.

Use of passive permeability data (Table 5) to mechanistically model the absorption of rifampicin did not capture the absorption kinetics of rifampicin. Very little data exists which supports the notion that rifampicin undergoes active transport, while the reported $\mathrm{Fa}$ of $>0.9{ }^{54}$ would support the notion that no active efflux transporter pathways exist which impact upon the oral bioavailability of rifampicin. However, to assess the impact of potential active efflux on rifampicin absorption, a sensitivity analysis was conducted where active intestinal efflux was attributed to ABCB1 ( $P$ glycoprotein) and the impact of variation in passive permeability $\left(\mathrm{P}_{\mathrm{app}}\right)$ and active efflux transporter clearance (CLtrans) on rifampicin Fa was assessed (Figure 5). Assuming rifampicin is a highly permeable compound (human jejunum effective permeability, $P_{\text {eff, }}>1 \times 10^{-4} \mathrm{~cm} / \mathrm{s}$; Simcyp predicted $P_{\text {eff: }} 2.15 \times 10^{-4} \mathrm{~cm} / \mathrm{s}$ ) (BCS Class II), active efflux would only impact upon rifampicin Fa under conditions of high efflux $(>10 \mu \mathrm{L} / \mathrm{min} / \mathrm{pmol})$. However, for our measured $\mathrm{P}_{\text {app }}\left(0.137 \times 10^{-6}\right.$ $\mathrm{cm} / \mathrm{s}$ ) the resultant $P_{\text {eff }}$ is $0.19 \times 10^{-4} \mathrm{~cm} / \mathrm{s}$ and would classify rifampicin as a low permeability compound. Furthermore, transporter clearance in excess of $1 \mu \mathrm{L} / \mathrm{min} / \mathrm{pmol}$ would impact upon the overall $\mathrm{Fa}$ of rifampicin. With this in mind, it was decided to utilise the default optimised rifampicin compound file (see Table 8) within Simcyp without alteration of the absorption kinetics.

Subsequent validation of isoniazid and rifampicin using validation data sets 3 and 4 for each API was successful and generally centred around the mean simulated profiles and were within the $5^{\text {th }}$ and $95^{\text {th }}$ percentiles of the simulated profiles (see Figures 6 and 7).

Simulations to predict the in vivo performance of ODTs in healthy volunteers were used to compare the bioavailability between single and FDC formulations under fasted and fed conditions, using the dissolution data. For isoniazid, the formulation state (single or combined) or dosing state (fasted or fed) had no statistically significant impact on pharmacokinetics (Figure $8 \mathrm{a}$ and b). Isoniazid plasma concentrations reached a geometric mean $\mathrm{C}_{\max }$ of $0.70-0.74 \mathrm{ng} / \mathrm{ml}$ in all conditions (Table 6), yielding a median AUC in the range of 4.05-4.24 ng/ml.h. 
1 At the level of the small-intestine Fa for isoniazid correlated with dissolution profiles, showing no significant differences between single and combination formulations, with values of $0.98 \pm 0.02$ and $0.97 \pm 0.03$ (fasted) and $0.99 \pm 0.04$ and $0.96 \pm 0.05$ (fed), respectively.

Fa values for rifampicin were equivalent between formulation states at 0.94 in fasted subjects; likewise, no difference was seen in Fa for fed subjects, with values of 0.94 for both single and combination doses. These results imply that permeation across the intestinal epithelial membrane was not rate limiting, casting doubt on the ability of $f_{1}$ and $f_{2}$ factor testing in this instance to predict bioequivalence. Rifampicin plasma profiles similarly showed no statistically significant difference $(p>0.05)$ in pharmacokinetic parameters between single and combination doses in fasted subjects (Figure 8c). Rifampicin plasma concentrations (Table 7) in FDCs (irrespective of dosing state) demonstrated higher AUCs $(9.26 \mathrm{ng} / \mathrm{ml} . \mathrm{h})$ compared to single formulations $(8.80 \mathrm{ng} / \mathrm{ml} . \mathrm{h})$. Furthermore, geometric mean $\mathrm{C}_{\max }$ was generally consistent across all formulations and conditions $(1.22-1.24 \mathrm{ng} / \mathrm{ml})$ with a $t_{\max }$ of $2.36-2.38 \mathrm{~h}$.

Bioavailability $(F)$ for isoniazid in all cases was 1 , whilst $F$ values for rifampicin were 0.91 . This may be related to the high Fa seen with both APIs. Bioavailability for rifampicin correlates well with reported values. Rifampicin is a CYP3A4 inducer ${ }^{55}$ and it is likely that over a longer study period (i.e. multidose over a few weeks) $\mathrm{F}$ would drop to around $65-70 \%$, as a result of increased metabolism ${ }^{54}$. Furthermore, due to the inclusion of ascorbic acid as an antioxidant and since dissolution and degradation was not tested in simulated gastric fluid (at a lower $\mathrm{pH}$ ), actual bioavailability values in vivo may differ. 


\section{Conclusion}

ODTs demonstrated satisfactory performance for hardness, friability and disintegration. Dissolution profile comparison between single and FDC formulations of isoniazid indicated bioequivalence regardless of dissolution media used and this was reinforced through PBPK modelling, with no difference in pharmacokinetic parameters. Comparable bioequivalence between single and FDC was not assumed for rifampicin from dissolution comparison in FeSSIF, with drug release falling by around $15 \%$, likely as a result of rifampicin degradation.

Clinical trial simulations reported no difference in isoniazid bioavailability between combination and single dose, despite isoniazid dissolution failing $\mathrm{f}_{2}$ testing in FaSSIF. Additionally, no food effect was seen. Notably also, the apparent decrease in rifampicin dissolution from FDCs in FeSSIF did not result in reduced bioavailability in fed subjects, whilst FDCs in fasted subjects similarly displayed bioequivalence with the single dose formulation, highlighting a failure in $f_{1}$ and $f_{2}$ factor testing.

PBPK modelling demonstrated that the bioavailability of either drug was unaltered as a result of combination with the other in these formulations. Rapid release isoniazid and rifampicin FDC ODTs thus may be a viable and attractive formulation prospect, whilst the framework used here could be employed in the development of more complex formulations. It should be noted that the focus for these investigations was on preformulation and initial dosage form development and therefore stability studies were not carried out.

The application of PBPK modelling in this study demonstrated the ability of this technique to predict in vivo performance based on in vitro experimental work and thus overcome the difficulties in performing clinical trials in paediatric populations. Although PBPK modelling cannot replace real-world clinical testing in paediatrics, with further studies in real paediatric populations being required to confirm the results seen here, PBPK offers a powerful tool to predict efficacy, safety and bioequivalence and aid in regulatory approval. 
1 Acknowledgement

2 Financial support for Tom Dennison was provided by a joint funded Medical Research Council

3 CASE award (Grant No MR/J01236X/1) with Viridian Pharma Ltd.

6

\section{Competing financial interests statement}

8 The authors report no competing financial interests regarding this work 


\section{References}

1 Daniel, T. M. The history of tuberculosis. Respiratory medicine 100, 18621870 (2006).

2 WHO. Global tuberculosis report. (World Health Organization, 2015).

3 Knechel, N. A. Tuberculosis: pathophysiology, clinical features, and diagnosis. Critical care nurse 29, 34-43 (2009).

4 Sharma, S. \& Mohan, A. Extrapulmonary tuberculosis. Indian Journal of Medical Research 120, 316 (2004).

5 Jensen, P. A. et al. Guidelines for preventing the transmission of Mycobacterium tuberculosis in health-care settings, 2005. (US Department of Health and Human Services, Public Health Service, Centers for Disease Control and Prevention, 2005).

6 Frieden, T. R., Sterling, T. R., Munsiff, S. S., Watt, C. J. \& Dye, C. Tuberculosis. The Lancet 362, 887-899, doi:http://dx.doi.org/10.1016/S01406736(03)14333-4 (2003).

7 Li, Y.-j., Petrofsky, M. \& Bermudez, L. E. Mycobacterium tuberculosis uptake by recipient host macrophages is influenced by environmental conditions in the granuloma of the infectious individual and is associated with impaired production of interleukin-12 and tumor necrosis factor alpha. Infection and immunity 70, 6223-6230 (2002).

8 Smith, I. Mycobacterium tuberculosis pathogenesis and molecular determinants of virulence. Clinical microbiology reviews 16, 463-496 (2003).

9 Feja, K. \& Saiman, L. Tuberculosis in children. Clinics in chest medicine 26, 295-312 (2005).

10 Peto, H. M., Pratt, R. H., Harrington, T. A., LoBue, P. A. \& Armstrong, L. R. Epidemiology of extrapulmonary tuberculosis in the United States, 19932006. Clinical Infectious Diseases 49, 1350-1357 (2009).

11 Narasimhan, P., Wood, J., MacIntyre, C. R. \& Mathai, D. Risk factors for tuberculosis. Pulmonary medicine 2013 (2013).

12 Corbett, E. L. et al. The growing burden of tuberculosis: global trends and interactions with the HIV epidemic. Archives of internal medicine 163, 1009-1021 (2003).

13 Cegielski, J. \& McMurray, D. The relationship between malnutrition and tuberculosis: evidence from studies in humans and experimental animals. The international journal of tuberculosis and lung disease 8, 286-298 (2004).

14 Muniyandi, M. et al. The prevalence of tuberculosis in different economic strata: a community survey from South India [Short Communication]. The International Journal of Tuberculosis and Lung Disease 11, 1042-1045 (2007).

15 Marais, B. \& Donald, P. The natural history of tuberculosis infection and disease in children. (2009).

16 WHO. General notes on Biopharmaceutics Classification System (BCS)-based biowaiver applications. Vol. 23 (World Health Organization, 2009).

17 Metcalfe, C. et al. The tuberculosis prodrug isoniazid bound to activating peroxidases. Journal of Biological Chemistry 283, 6193-6200 (2008). 
18 Somoskovi, A., Parsons, L. M. \& Salfinger, M. The molecular basis of 2 resistance to isoniazid, rifampin, and pyrazinamide in Mycobacterium

tuberculosis. Respiratory research 2, 1 (2001).

Becker, C. et al. Biowaiver monographs for immediate release solid oral dosage forms: Rifampicin. Journal of pharmaceutical sciences 98, 22522267 (2009).

20 Hartmann, G., Honikel, K. O., Knüsel, F. \& Nüesch, J. The specific inhibition of the DNA-directed RNA synthesis by rifamycin. Biochimica et Biophysica Acta (BBA)-Nucleic Acids and Protein Synthesis 145, 843-844 (1967).

21 Campbell, E. A. et al. Structural mechanism for rifampicin inhibition of bacterial RNA polymerase. Cell 104, 901-912 (2001).

22 Gohel, M. C. \& Sarvaiya, K. G. A novel solid dosage form of rifampicin and isoniazid with improved functionality. AAPS PharmSciTech 8, E133-E139 (2007).

23 WHO. Treatment of tuberculosis: guidelines. (World Health Organization, 2010).

24 Gallardo, C. R. et al. Fixed - dose combinations of drugs versus single drug formulations for treating pulmonary tuberculosis. The Cochrane Library (2016).

25 Albanna, A. S., Smith, B. M., Cowan, D. \& Menzies, D. Fixed-dose combination antituberculosis therapy: a systematic review and meta-analysis. European Respiratory Journal 42, 721-732 (2013).

26 Sastry, S. V., Nyshadham, J. R. \& Fix, J. A. Recent technological advances in oral drug delivery-a review. Pharmaceutical science \& technology today $\mathbf{3}$, 138-145 (2000).

27 Hirani, J. J., Rathod, D. A. \& Vadalia, K. R. Orally disintegrating tablets: A review. Tropical Journal of Pharmaceutical Research 8 (2009).

28 Use, C. f. M. P. f. H. \& Use, C. f. M. P. f. H. Guideline on the investigation of bioequivalence. European Medicines Agency (EMA), London (2010).

29 Food \& Administration, D. Guidance for industry: bioavailability and bioequivalence studies submitted in NDAs or INDs-General Considerations. Rockville, MD: Food and Drug Administration (2014).

30 Yang, S.-G. Biowaiver extension potential and IVIVC for BCS Class II drugs by formulation design: Case study for cyclosporine self-microemulsifying formulation. Archives of pharmacal research 33, 1835-1842 (2010).

31 Kostewicz, E. S. et al. PBPK models for the prediction of in vivo performance of oral dosage forms. European Journal of Pharmaceutical Sciences 57, 300321 (2014).

32 Badhan, R. K. S. Physiologically Based Pharmacokinetic Modelling in Drug Delivery. Computational Pharmaceutics: Application of Molecular Modeling in Drug Delivery, 263 (2015).

33 Jamei, M. et al. Population-based mechanistic prediction of oral drug absorption. The AAPS journal 11, 225-237 (2009).

34 Kesisoglou, F. \& Mitra, A. Application of absorption modeling in rational design of drug product under quality-by-design paradigm. The AAPS journal 17, 1224-1236 (2015).

35 Crison, J. R. et al. Biowaiver approach for biopharmaceutics classification system class 3 compound metformin hydrochloride using in silico modeling. Journal of pharmaceutical sciences 101, 1773-1782 (2012). 
36 McLaughlin, R., Banbury, S. \& Crowley, K. Orally disintegrating tablets: the

2 effect of recent FDA guidance on ODT technologies and applications.

37 Connor, J. in Fixed-dose combinations for HIV/AIDS, tuberculosis and malaria World Health Organisation 119 (2003).

38 Conte, J. E., Lin, E. \& Zurlinden, E. Liquid chromatographic determination of rifampin in human plasma, bronchoalveolar lavage fluid, and alveolar cells. Journal of chromatographic science 38, 72-76 (2000).

39 Klancke, J. Dissolution testing of orally disintegrating tablets. Dissolution technologies 10, 6-9 (2003).

40 Ranaldi, G., Islam, K. \& Sambuy, Y. Epithelial cells in culture as a model for the intestinal transport of antimicrobial agents. Antimicrobial agents and chemotherapy 36, 1374-1381 (1992).

41 Howgate, E. M., Rowland Yeo, K., Proctor, N. J., Tucker, G. T. \& RostamiHodjegan, A. Prediction of in vivo drug clearance from in vitro data. I: impact of inter-individual variability. Xenobiotica; the fate of foreign compounds in biological systems 36, 473-497, doi:10.1080/00498250600683197 (2006).

42 Jamei, M. et al. Population-based mechanistic prediction of oral drug absorption. Aaps j 11, 225-237, doi:10.1208/s12248-009-9099-y (2009).

43 Hao, L. H. et al. Comparative bioavailability of rifampicin and isoniazid in fixed-dose combinations and single-drug formulations. The International Journal of Tuberculosis and Lung Disease 18, 1505-1512, doi:10.5588/ijtld.13.0647 (2014).

44 Agrawal, S. et al. Comparative bioavailability of rifampicin, isoniazid and pyrazinamide from a four drug fixed dose combination with separate formulations at the same dose levels. International Journal of Pharmaceutics 276, 41-49, doi:http://dx.doi.org/10.1016/j.ijpharm.2004.02.019 (2004).

45 Milán-Segovia, R. C. et al. Relative bioavailability of isoniazid in a fixed-dose combination product in healthy Mexican subjects. The International Journal of Tuberculosis and Lung Disease 18, 49-54, doi:10.5588/ijtld.13.0266 (2014).

$46 \mathrm{Xu}$, J. et al. Oral Bioavailability of Rifampicin, Isoniazid, Ethambutol, and Pyrazinamide in a 4-Drug Fixed-Dose Combination Compared With the Separate Formulations in Healthy Chinese Male Volunteers. Clinical Therapeutics 35, 161-168, doi:http://dx.doi.org/10.1016/j.clinthera.2013.01.003 (2013).

Rohatgi, A. WebPlotDigitizer 3.10. See http://arohatgi.

48 FDA, U. Guidance for Industry: Dissolution testing of immediate-release solid oral dosage forms. Food and Drug Administration, Center for Drug Evaluation and Research (CDER) (1997).

49 Food \& Administration, D.

50 Thoorens, G., Krier, F., Leclercq, B., Carlin, B. \& Evrard, B. Microcrystalline cellulose, a direct compression binder in a quality by design environment-A review. International journal of pharmaceutics 473, 6472, doi:http://dx.doi.org/10.1016/j.ijpharm.2014.06.055 (2014). 
51 Saigal, N., Baboota, S., Ahuja, A. \& Ali, J. Microcrystalline cellulose as a 2 versatile excipient in drug research. Journal of Young Pharmacists 1, 6 3 (2009).

452 Westermarck, S., Juppo, A. M., Kervinen, L. \& Yliruusi, J. Microcrystalline cellulose and its microstructure in pharmaceutical processing. European journal of pharmaceutics and biopharmaceutics 48, 199-206 (1999).

53 Lakshminarayana, S. B. et al. Comprehensive physicochemical, pharmacokinetic and activity profiling of anti-TB agents. Journal of Antimicrobial Chemotherapy 70, 857-867 (2015).

54 Loos, U. et al. Pharmacokinetics of oral and intravenous rifampicin during chronic administration. Klinische Wochenschrift 63, 1205-1211 (1985).

55 Baneyx, G., Parrott, N., Meille, C., Iliadis, A. \& Lavé, T. Physiologically based pharmacokinetic modeling of CYP3A4 induction by rifampicin in human: influence of time between substrate and inducer administration. European Journal of Pharmaceutical Sciences 56, 1-15 (2014). 


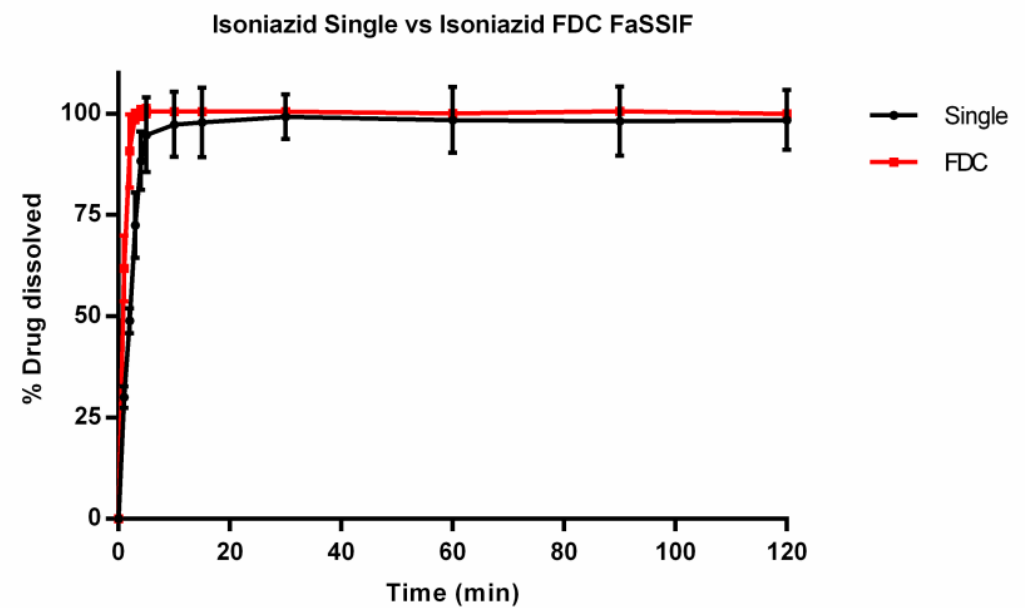

Figure 1. Isoniazid $(50 \mathrm{mg})$ dissolution profiles of single and FDC formulations in fasted state biorelevant media $\left(900 \mathrm{ml}, 37^{\circ} \mathrm{C}\right)$ from $500 \mathrm{mg}$ ODTs. Dissolution performed using USP 2 paddle apparatus (mean $\pm S D, n=3$ ) 


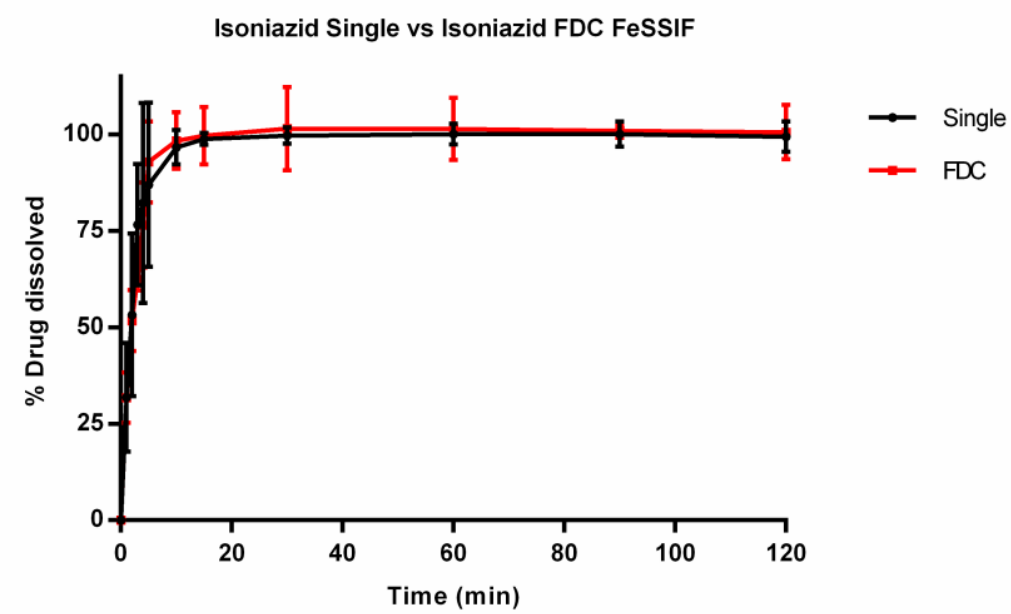

Figure 2. Isoniazid $(50 \mathrm{mg}$ ) dissolution profiles of single and FDC formulations in fed state biorelevant media $\left(900 \mathrm{ml}, 37^{\circ} \mathrm{C}\right)$ from $500 \mathrm{mg}$ ODTs. Dissolution performed using USP 2 paddle apparatus (mean $\pm S D, n=3$ ) 


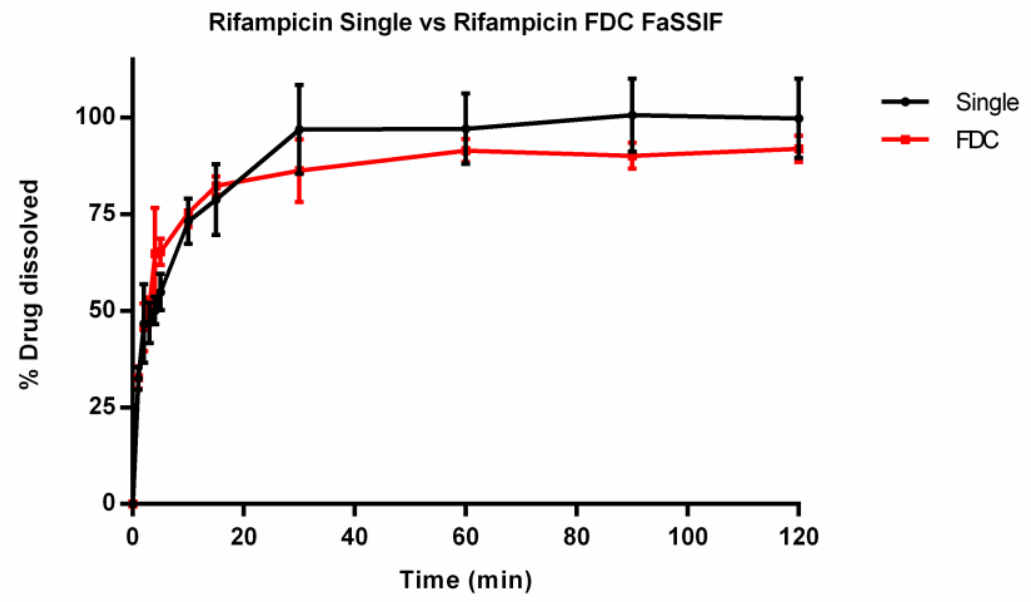

Figure 3. Rifampicin $(75 \mathrm{mg}$ ) dissolution profiles of single and FDC formulations in fasted state biorelevant media $\left(900 \mathrm{ml}, 37^{\circ} \mathrm{C}\right)$ from $500 \mathrm{mg}$ ODTs. Dissolution performed using USP 2 paddle apparatus (mean $\pm S D, n=3$ ) 


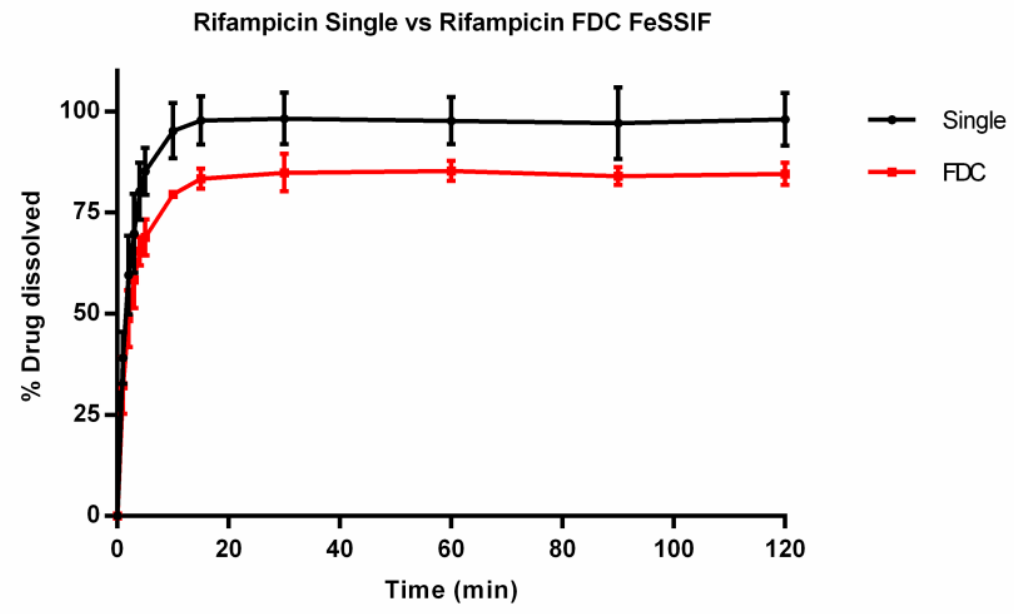

Figure 4. Rifampicin $(75 \mathrm{mg})$ dissolution profiles of single and FDC formulations in fed state biorelevant media $\left(900 \mathrm{ml}, 37^{\circ} \mathrm{C}\right)$ from $500 \mathrm{mg}$ ODTs. Dissolution performed using USP 2 paddle apparatus (mean $\pm \mathrm{SD}, \mathrm{n}=3$ ) 


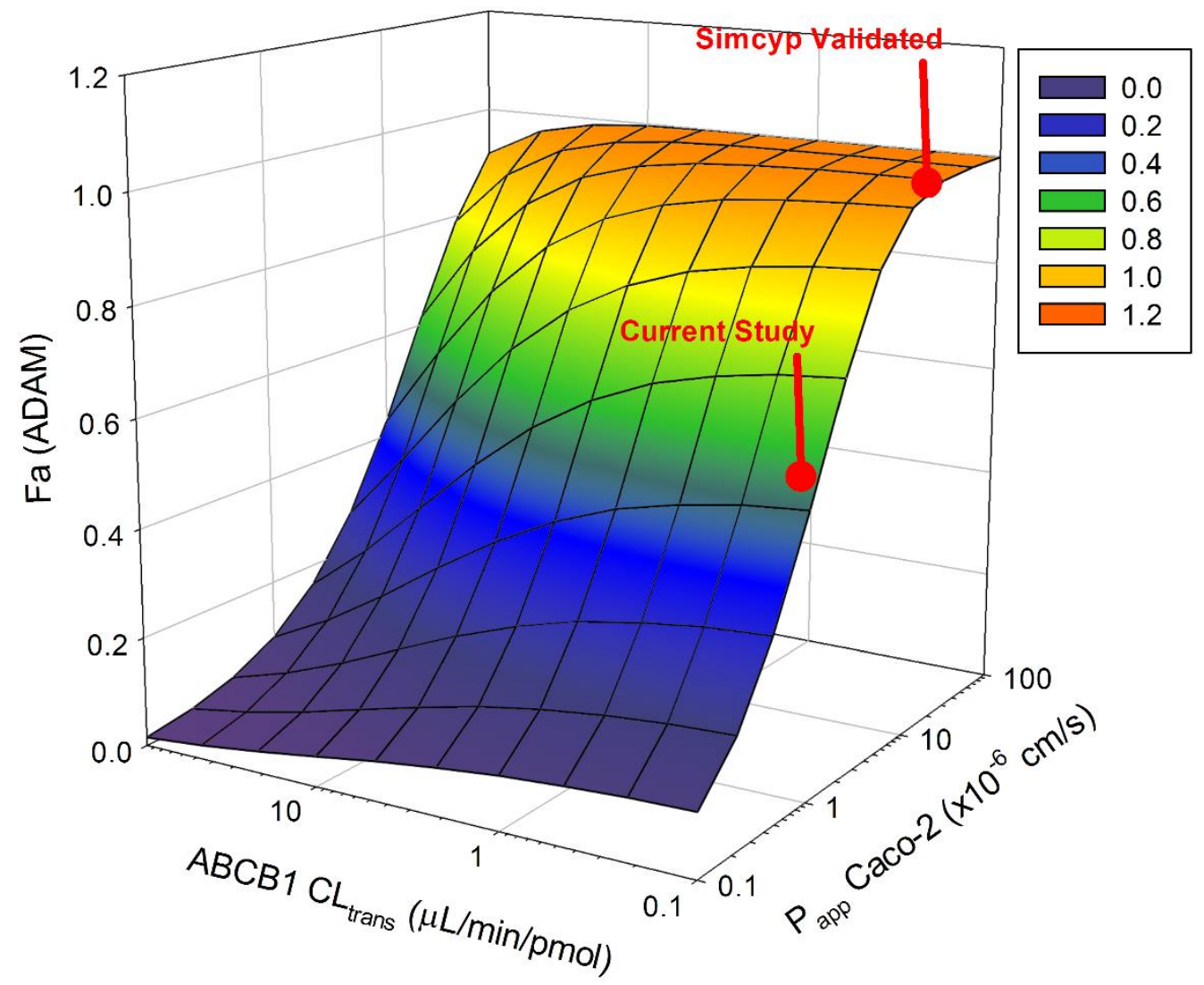

Figure 5. Sensitivity analysis of rifampicin (Simcyp default compound) fraction dose absorbed (fa) when varying $P_{\text {app }}\left(0.1-100 \times 10^{-6} \mathrm{~cm} / \mathrm{s}\right)$ and intestinal active efflux (CL trans) $\left(0.1-100 \mu \mathrm{L} / \mathrm{min} / \mathrm{pmol}\right.$ ABCB1). $P_{\text {app }}$ values for the current study and those validated by Simcyp are highlighted. 


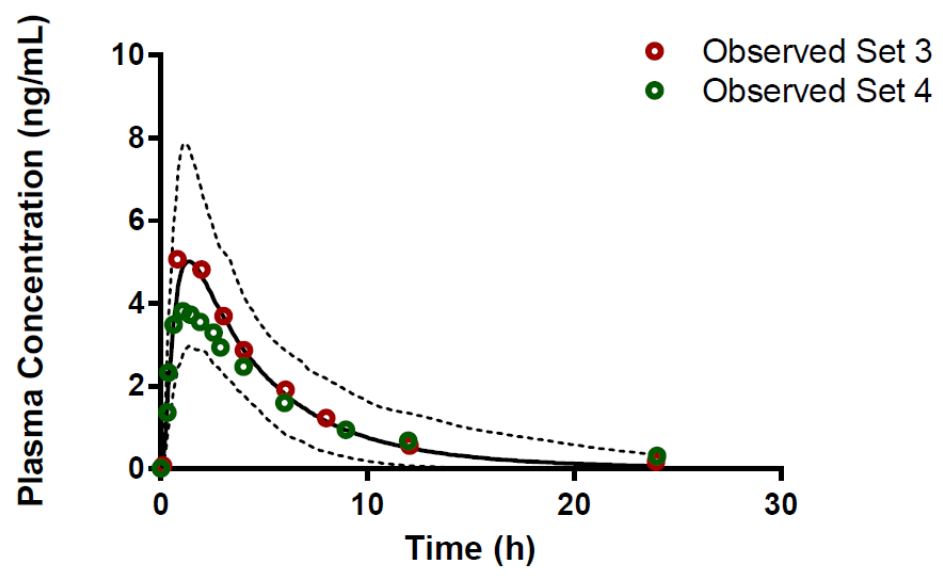

Figure 6. Simulated mean plasma profile after a $300 \mathrm{mg}$ oral dose of isoniazid (solid black line). The corresponding observed data points are shown by red open circles. The grey lines represent the 5th and 95th percentiles for the predicted values. All simulations were performed using the minimal PBPK model. 


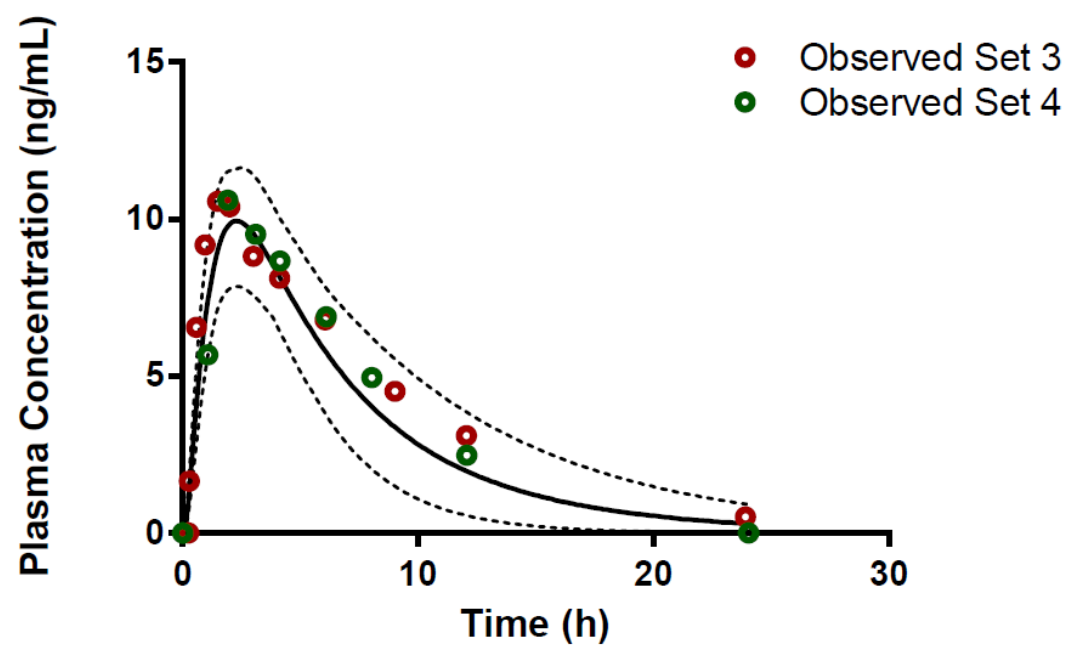

Figure 7. Simulated mean plasma profile after a $600 \mathrm{mg}$ oral dose of rifampicin (solid black line). The corresponding observed data points are shown by red (set 3) or green (set 4) open circles. The grey lines represent the 5th and 95th percentiles for the predicted values. All simulations were performed using the minimal PBPK model. 
(a)

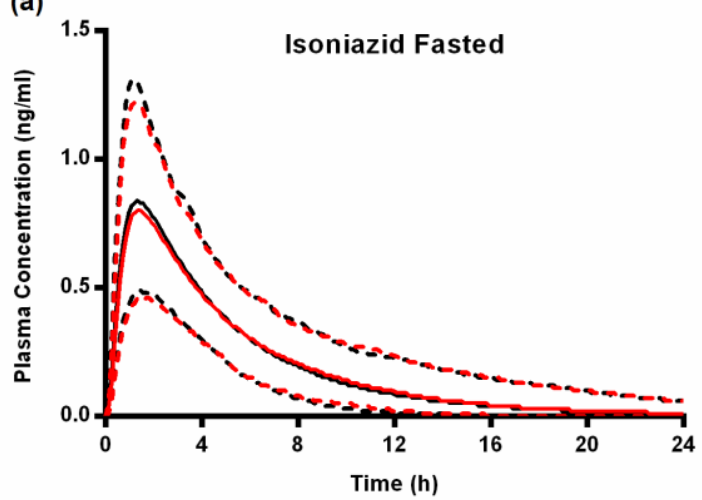

(c)

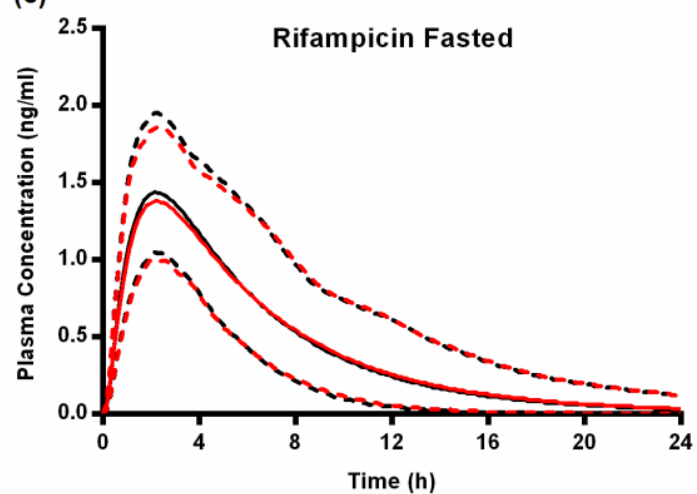

(b)

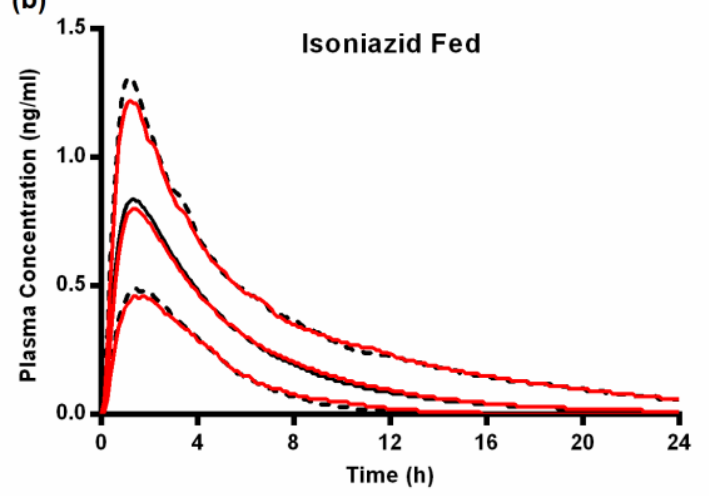

(d)

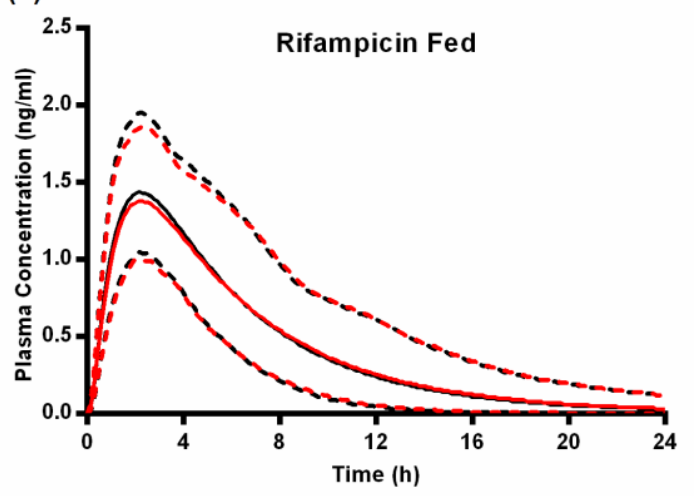

Figure 8. Simulated mean plasma profile after a $50 \mathrm{mg}$ oral dose of isoniazid (a and b) and $75 \mathrm{mg}$ oral dose of rifampicin (c and d) under fasted and fed conditions. Single API formulations indicated in black and fixed-dose combination in red. Solid lines represent trial mean and dashed lines represent the $5^{\text {th }}$ and $95^{\text {th }}$ percentiles for the predicted values. 
Table 1. ODT formulations for individual dose and FDC ODTs. Values for APIs and excipients are given as $\% \mathrm{w} / \mathrm{w}$ for $500 \mathrm{mg}$ tablets. All formulations underwent compaction at $2.2 \mathrm{~T}$ with a $6 \mathrm{~s}$ dwell time.

\begin{tabular}{cccc}
\hline & Isoniazid (10\%) & Rifampicin (15\%) & $\begin{array}{c}\text { Isoniazid + Rifampicin } \\
(\mathbf{1 0 \% + 1 5 \% )}\end{array}$ \\
\hline Isoniazid & F1 & F2 & F3 \\
Rifampicin & 50 & 75 & 50 \\
Pearlitol & & 342.5 & 75 \\
Flash & 367.5 & 7.5 & 292.5 \\
SSF (1.5\%) & 7.5 & 75 & 7.5 \\
MCC (15\%) & 75 & & 75 \\
\hline
\end{tabular}


Table 2. Individual and FDC ODT properties. All formulations underwent compaction at $2.2 \mathrm{~T}$ with a 6 s dwell time.

\begin{tabular}{ccccc}
\hline Hardness (N) & Porosity & $\begin{array}{c}\text { Disintegration Time } \\
\text { (s) }\end{array}$ & $\begin{array}{c}\text { Friability (\% weight } \\
\text { loss) }\end{array}$ \\
\hline F1 & $95.50 \pm 1.15$ & $0.26 \pm 0.01$ & $22.67 \pm 1.53$ & 1.10 \\
F2 & $143.90 \pm 15.47$ & $0.25 \pm 0.01$ & $22.67 \pm 1.15$ & 0.86 \\
F3 & $151.17 \pm 4.48$ & $0.23 \pm 0.01$ & $26.67 \pm 2.52$ & 0.85 \\
\hline
\end{tabular}


Table 3. HPLC method validation for detection of isoniazid and rifampicin both alone and in combination. Data for linearity (correlation coefficient), instrument precision, accuracy (recovery), precision (\% RSD), LOD and LOQ are displayed

\begin{tabular}{lcccccc}
\hline & $\begin{array}{c}\text { Instrument precision } \\
(\% \text { RSD) }\end{array}$ & $\begin{array}{c}\text { Recovery (mean } \\
\% \pm \text { SD) }\end{array}$ & $\begin{array}{c}\text { Intraday precision } \\
(\text { mean \% RSD) }\end{array}$ & LOD $(\mu \mathrm{g} / \mathbf{m l})$ & LOQ ( $\mu \mathrm{g} / \mathrm{ml})$ & $\begin{array}{c}\text { Correlation } \\
\text { coefficient }\end{array}$ \\
\hline Isoniazid & 0.08 & $99.53 \pm 0.60$ & 0.88 & 0.24 & 0.80 & 0.99997 \\
Rifampicin & 0.13 & $100.33 \pm 1.13$ & 2.07 & 0.14 & 0.46 & 0.99994 \\
Isoniazid combination & 0.27 & $99.64 \pm 1.06$ & 1.47 & 0.15 & 0.51 & 0.99996 \\
Rifampicin combination & 0.87 & $100.49 \pm 1.28$ & 1.20 & 0.36 & 1.18 & 0.99987 \\
\hline
\end{tabular}


Table 4. Comparison of dissolution profiles for each compound from single and FDC formulations in FaSSIF and FeSSIF media, by difference factor $f_{1}$ and similarity factor $f_{2}$ testing. Dissolution profiles are considered similar if the $f_{1}$ value is below 15 and the $f_{2}$ value is above 50 .

\begin{tabular}{ccccc}
\hline Compound & & $\mathbf{8} \mathbf{8 5} \%$ Dissolution $\mathbf{5}$ 15 $\mathbf{m i n}$ & $\mathbf{f}_{\mathbf{1}}$ & $\mathbf{f}_{\mathbf{2}}$ \\
\hline \multirow{2}{*}{ Isoniazid } & FaSSIF & Yes & 14.17 & 32.79 \\
& FeSSIF & Yes & 3.78 & 65.30 \\
\multirow{2}{*}{ Rifampicin } & FaSSIF & No & 9.30 & 55.76 \\
& FeSSIF & No & 15.55 & 44.82 \\
\hline
\end{tabular}


Table 5. $\mathrm{P}_{\text {app }}$ values for isoniazid and rifampicin alone and in combination in A-B and B-A directions, across Caco-2 monolayers at $\mathrm{pH} 7.4$ in both compartments (mean $\pm \mathrm{SD}, \mathrm{n}=3$ )

\begin{tabular}{cccc}
\hline Compound & \multicolumn{2}{c}{$\mathbf{P a p p}_{\text {10 }} \mathbf{1 0}^{-6} \mathbf{c m ~ s}^{-1}$} & Efflux Ratio \\
\hline Isoniazid & A-B & B-A & \\
Rifampicin & $16.37 \pm 0.48$ & $19.27 \pm 0.32$ & 1.18 \\
Isoniazid Combination & $1.37 \pm 0.12$ & $5.95 \pm 0.42$ & 4.33 \\
Rifampicin Combination & $22.69 \pm 1.21$ & $26.98 \pm 0.26$ & 1.19 \\
\hline
\end{tabular}


Table 6. Summary of pharmacokinetic parameters for isoniazid $(50 \mathrm{mg})$ under fasted and fed conditions. Geometric mean (SD) reported for $\mathrm{C}_{\max }$ and median (range) for AUC and $t_{\max }$

\begin{tabular}{rcccc}
\hline & \multicolumn{2}{c}{ Isoniazid Fasted } & \multicolumn{2}{c}{ Isoniazid Fed } \\
\cline { 2 - 5 } Parameters & Single & Combined & Single & Combined \\
\hline AUC $(\mathbf{n g} / \mathbf{m l} . \mathbf{h})$ & $4.05(3.14-7.10)$ & $4.24(3.13-7.41)$ & $4.05(3.14-7.10)$ & $4.24(3.13-7.42)$ \\
$\mathbf{C}_{\max }(\mathbf{n g} / \mathbf{m l})$ & $0.74(0.13)$ & $0.70(0.12)$ & $0.74(0.13)$ & $0.70(0.12)$ \\
$t_{\max }(\mathbf{h})$ & $1.48(1.14-1.92)$ & $1.49(1.21-1.96)$ & $1.48(1.14-1.92)$ & $1.49(1.12-1.91)$ \\
\hline
\end{tabular}


Table 7. Summary of pharmacokinetic parameters for rifampicin $(75 \mathrm{mg})$ under fasted and fed conditions. Geometric mean (SD) reported for $\mathrm{C}_{\mathrm{max}}$ and median (range) for AUC and tmax

\begin{tabular}{rcccc}
\hline \multirow{2}{*}{ Parameters } & \multicolumn{2}{c}{ Rifampicin Fasted } & \multicolumn{2}{c}{ Rifampicin Fed } \\
\cline { 2 - 5 } & Single & Combined & Single & Combined \\
\hline AUC $(\mathbf{n g} / \mathbf{m l} . \mathbf{h})$ & $8.80(6.63-13.63)$ & $9.26(6.61-13.50)$ & $1.24(0.63-13.63)$ & $9.26(6.61-13.50)$ \\
$\mathbf{C}_{\max }(\mathbf{n g} / \mathrm{ml})$ & $1.24(0.18)$ & $1.22(0.30)$ & $2.38(1.51-2.80)$ & $1.22(0.30)$ \\
$t_{\max }(\mathrm{h})$ & $2.38(1.51-2.80)$ & $2.38(1.80-2.85)$ & $2.36(1.80-2.86)$ & \\
\hline
\end{tabular}


Table 8 Input parameter values and predicted PBPK values for simulation of pharmacokinetics of isoniazid and rifampicin.

\begin{tabular}{ccc}
\hline Parameter & Isoniazid & Rifampicin \\
\hline Type & Monoprotic base & Ampholyte \\
MW & 137.1 & 823 \\
LogP & -0.7 & 4.01 \\
pKa & 1.82 & $1.7,7.9$ \\
fu & 0.95 & 0.113 \\
Vss (L/kg) & Predicted PBPK/PE & 0.42 (Full PBPK) \\
B:P ratio & 0.825 & 0.9 \\
Clpo (L/min) & 12 & 8.75 \\
Peff (cms/s) & PE & 2.15 \\
\hline
\end{tabular}

MW: molecular weight; fu: plasma unbound fraction; Vss: steady-state volume of distribution; B:P ratio: blood-to-plasma ratio; CLpo: oral clearance; Peff: human effective permeability. ${ }^{a}$ Vss was determined from calculation of tissue partitions coefficients within Simcyp or parameter estimated (PE). 